\title{
On the ethics of social egg freezing and fertility preservation for nonmedical reasons
}

This article was published in the following Dove Press journal:

Medicolegal and Bioethics

19 August 2015

Number of times this article has been viewed

\section{Karey A Harwood}

Department of Philosophy and Religious Studies, North Carolina State University, Raleigh, NC, USA

Correspondence: Karey A Harwood Department of Philosophy and Religious Studies, North Carolina State University, I0I Lampe Drive, 340 Withers Hall Campus Box 8103, Raleigh,

NC 27695-8I03, USA

$\mathrm{Tel}+19195156383$

$\mathrm{Fax}+19195134351$

Email karey_harwood@ncsu.edu
Abstract: The practice of egg freezing reached a new milestone in 2012, when the American Society for Reproductive Medicine removed its designation as "experimental". Studies of the safety and efficacy of egg freezing led the ASRM to recommend egg freezing for patients facing infertility due to gonadotoxic therapies, but prompted continued caution against egg freezing when undertaken for nonmedical reasons. The European Society of Human Reproduction and Embryology has more explicitly supported nonmedical egg freezing. Ethical debate about nonmedical egg freezing raises many familiar issues, including the limits of individual autonomy when a medical technology is used for an elective reason. Concerns include commercial exploitation, pressure on women to use egg freezing, and the overall impact of egg freezing on sex inequality and professional norms. The ethical debate also calls for a more careful consideration of whether age-related fertility decline should count as a medical justification for fertility preservation. In lieu of broad consensus on these matters, this paper recommends honoring the principle of autonomy while insisting on better information about utilization and outcomes. Given the significant drop-off in success rates for women who attempt egg freezing when they are older than 38 years, full disclosure in the informed consent process must involve information about success rates by age-group. Much greater consistency and thoroughness in reporting would yield better and more generalizable data.

Keywords: egg freezing, ethics, delayed childbearing, assisted reproductive technology, infertility

\section{Introduction}

"Egg freezing" and "egg banking" are common terms for the cryopreservation and storage of human oocytes for later personal use or donation. While the two processes parallel sperm freezing and sperm banking, both the science and the ethics of egg freezing have lagged behind the well-established practice of freezing sperm. Due to their larger size, higher water content, and specific chromosomal arrangement, human eggs have required the development of more sophisticated techniques for successful freezing, and data about outcomes only recently reached a critical threshold.

In 2012, in a reversal of its previous position, the American Society for Reproductive Medicine (ASRM) declared that egg freezing should no longer be considered experimental. ${ }^{1}$ Although far from a blanket endorsement of egg freezing, the ASRM's updated position represents a significant step in the evolution of society's views about this controversial technology. The ASRM based its statement on evidence comparing the fertilization and pregnancy rates of fresh versus frozen eggs, as well as evidence about the safety of egg freezing. From 80 studies of cryopreservation efficacy and 32 studies 
of cryopreservation safety, the ASRM concluded that enough evidence existed to justify removing the label of "experimental" from egg freezing, but not enough to recommend its use for universal donor egg banking or "for the sole purpose of circumventing reproductive aging in healthy women". ${ }^{1}$ The ASRM only explicitly recommended egg freezing for women facing infertility "due to chemotherapy or other gonadotoxic therapies". ${ }^{1}$ Indeed, the ASRM underscored the limited nature of available data and emphasized the need for ongoing research. Also noteworthy was the conclusion that success rates appeared to be significantly lower for women who freeze their eggs over the age of 38 years.

This partial endorsement of egg freezing by an authoritative medical society unquestionably influences the ethical debate about this reproductive technology. ${ }^{2,3}$ The split recommendation - recommending egg freezing for medical reasons, but not yet for nonmedical reasons - invites a more careful analysis of the moral distinction between medical and nonmedical reasons for egg freezing. ${ }^{4}$ The fact that success rates for egg freezing vary significantly based on the age of the woman at the time she freezes her eggs also raises the question of whether different ethical guidelines ought to be applied to different age-groups. ${ }^{5}$ Finally, lifting the experimental label, despite clear notes of caution, carries symbolic weight, effectively legitimizing egg freezing and potentially expanding its market. These consequences warrant further ethical analysis.

In the second decade of the twenty-first century, a majority of US fertility clinics offer egg freezing as one of their services, and most programs offer egg freezing for both medical and nonmedical reasons. ${ }^{6}$ Commercial egg banks (CEBs) also make frozen donor eggs widely available. ${ }^{7}$ Analogous to sperm banks, CEBs store and make available donated eggs for individuals seeking to create an embryo through in vitro fertilization (IVF). For example, women who are pursuing IVF with donor eggs might prefer to use frozen rather than fresh eggs to control the timing of fertilization and implantation. According to a 2013 study, frozen donor eggs comprise only a minority of donor-egg IVF cycles, although their prevalence is expected to increase with the establishment of more CEBs. ${ }^{7}$

A large part of the increased confidence in egg freezing is due to the development of the vitrification technique, a procedure that quickly freezes the egg using extreme cooling rates. ${ }^{8}$ This method avoids the formation of crystals inside the egg, which can cause cellular damage, and has resulted in high rates of egg survival, fertilization, embryo development, and pregnancy. Although there are small variations in this method, most clinics use vitrification rather than the older slow-freezing technique. Another advance credited for improving the success rates of egg freezing is the combination of intracytoplasmic sperm injection (ICSI) with IVF when the time comes to thaw eggs and attempt fertilization and implantation. ICSI is the injection of an individual sperm directly into the egg. Because the zona pellucida of the egg hardens during freezing, ICSI can overcome what might otherwise be an insurmountable barrier to fertilization.

This paper identifies the commonly advanced medical and nonmedical reasons for egg freezing, and illustrates how a bright line between these two types of reasons is difficult to draw. It also surveys the ethical arguments in favor of and against egg freezing, paying special attention to nonmedical reasons, and concludes with a discussion of avenues to professional acceptance and effective policies.

\section{Medical reasons}

Cancer is among the most commonly cited medical reasons for seeking egg freezing. ${ }^{9,10}$ Survival rates of many cancers have improved, making it possible to plan for a life after cancer. For example, the 5-year survival rate for women with breast cancer is nearly $90 \% .{ }^{11}$ However, many treatments for cancer also impair fertility. Chemotherapy and radiation are both gonadotoxic therapies in that they may damage the ovaries. According to the ASRM, "Ovarian damage is drugand dose-dependent and is related to the age at the time of treatment, with progressively smaller doses producing ovarian failure as the patient's age increases."12 If a woman's specific cancer diagnosis and treatment plan afford her adequate opportunity, she may choose to undergo ovarian stimulation and egg retrieval in order to freeze her eggs before undergoing cancer treatment. In fact, given that egg freezing is no longer considered experimental, the American Society of Clinical Oncology now recommends that health care providers discuss the cryopreservation of unfertilized oocytes as an option for preserving fertility in female patients diagnosed with cancer. ${ }^{10}$ In its guidelines for health care providers, egg freezing is specifically labeled "standard practice" alongside sperm and embryo freezing. ${ }^{10}$

In addition to the freezing and banking of individual eggs, fertility preservation can be accomplished through the banking of ovarian tissue. In this case, ovarian tissue is surgically removed, cryopreserved, and thawed/reimplanted at a later time. For some, including pediatric cancer patients or adult women whose cancer diagnosis and treatment plan make ovarian stimulation and egg retrieval impossible, the option of ovarian tissue banking can be the only means of preserving 
future fertility. Unlike egg freezing, the cryopreservation of ovarian tissue is still considered experimental. ${ }^{12,13}$

Cancer is not the only medical reason that might motivate women to pursue egg freezing. There are a number of noncancerous conditions that can also justify fertility preservation. For example, some autoimmune diseases and hematological diseases are treated with chemotherapy, and thus involve the same risks to future fertility. ${ }^{12}$ Premature menopause does not involve gonadotoxic therapies, and is not itself a life-threatening condition, but it does cause an abnormally early end to a woman's reproductive years. A woman at risk for premature menopause (or for premature ovarian failure due to a number of genetic conditions) might be motivated to freeze her eggs.

Similarly, inheriting a mutation in the $B R C A 1$ or $B R C A 2$ genes is not in itself a disease, but it does bring a significantly increased risk of breast and/or ovarian cancer. Even without a cancer diagnosis, a woman who discovers she has a $B R C A$ mutation might choose to undergo a prophylactic oophorectomy to decrease her risk of cancer. Egg retrieval and egg freezing in advance of the removal of her ovaries can be a means of preserving potential future fertility. ${ }^{1}$

A final group of medical reasons for egg freezing involves the medical limitations of a couple seeking to become pregnant through IVF. For example, a male partner may be unable to produce a semen sample. If the woman has undergone egg retrieval for the purpose of IVF and a semen sample is not readily available, IVF cannot proceed. ${ }^{1}$ Egg freezing affords more time for the procurement of needed sperm. A couple might also be unwilling to cryopreserve embryos, perhaps for ethical or religious reasons. In this case, their choice to cryopreserve unfertilized eggs rather than embryos is not truly motivated by medical reasons, but by nonmedical preferences.

Egg freezing in advance of gonadotoxic chemotherapy is not precisely analogous to egg freezing for a healthy woman undergoing IVF whose male partner is unable to produce a semen sample. Nevertheless, both examples are more accurately characterized as being medically grounded reasons for egg freezing, as opposed to nonmedical reasons for egg freezing, provided infertility is considered a disease like any other. If a male partner cannot produce sperm, it is the couple that are experiencing infertility, even if the female partner has a healthy reproductive system.

\section{Nonmedical reasons}

Nonmedical reasons for egg freezing can be as varied as the women who consider undergoing the procedure.
Nonmedical egg freezing is also referred to as "elective" or "social" egg freezing in the literature, although "nonmedical egg freezing" is arguably a more accurate description since there are elective and social aspects to all egg freezing.

The most frequently cited reason for nonmedical egg freezing is lack of a suitable partner, sometimes combined with concern about advancing age..$^{3,14,15}$ Other reasons include a desire to postpone childbearing while completing one's education or while focusing on career advancement. Still other reasons include a desire to postpone childbearing until women feel they have reached a sufficient level of maturity, financial stability, or emotional support. A hyperfocus on individual women's decisions to postpone childbearing is misleading, however, if it neglects social context. For a variety of reasons, a steady trend toward delayed childbearing across all subgroups of women is a social reality. ${ }^{16}$ Women who find themselves in their late 30 s and involuntarily childless are in some respects merely experiencing the consequences of dutifully following the social and professional scripts laid out for them: to become educated, to contribute to the economy, and to prioritize work over family.

Some scholars identify the avoidance of age-related fertility decline - characterized by the ASRM as the "circumventing" of reproductive aging in healthy women - as a nonmedical reason for egg freezing. However, the tide may be turning toward conceptualizing age-related fertility loss as a medical reason for egg freezing. In some of the literature, egg freezing is characterized as "preventive medicine" for age-related fertility decline. The European Society of Human Reproduction and Embryology, for example, makes the case that fertility preservation for natural ovarian aging "cannot so easily be dismissed as a non-health-related preference." ${ }^{15}$ In its view, the morally relevant point is that the childlessness is involuntary, regardless of its cause. Indeed, the issue of age-related fertility decline exemplifies how difficult it is to draw a meaningful line between medical and nonmedical reasons for egg freezing.

In a related example, some researchers have pointed to the possible endocrine benefits of fertility preservation for postmenopausal women. As women live longer, they may not wish to spend a large portion of their adult years in menopause, or so researchers hypothesize. In this case, fertility preservation is accomplished by ovarian tissue freezing, and the goal is not necessarily to have a child. The goal is to rejuvenate youthfulness. Because menopause is not an illness, this motivation is difficult to classify as either clearly medical or clearly nonmedical. Nevertheless, the fact that aging is a natural process does not obviate the theoretical 
health benefits to older women if the cryopreserved ovarian tissue were successfully reimplanted. ${ }^{17}$

Finally, egg freezing can play a role in enabling childbearing for gays, lesbians, and unmarried persons. For example, a gay male couple could procure a frozen donor egg and the services of a surrogate mother in order to complete IVF. A lesbian couple might freeze their eggs while searching for donor sperm. As society moves closer to accepting a universal human interest in reproducing that is not confined to a heterosexual norm, it becomes more difficult to justify the denial of access to assisted reproductive technologies on the basis of sexual orientation and/or marital status. In a 2013 statement by its ethics committee, the ASRM called for programs providing fertility services to "treat all requests for assisted reproduction equally without regard to marital/ partner status or sexual orientation."18 They supported their recommendation with research that suggests children are not harmed in their development by being raised by same-sex parents. They also noted that claims of physician autonomy or religious freedom are not legitimate bases for discrimination on the basis of sexual orientation. ${ }^{18}$

To attempt to delineate the nonmedical reasons for egg freezing is already to begin an ethical analysis of what counts as a valid reason for egg freezing. Discussing how egg freezing is paid for similarly complicates our common understandings of health and illness, necessity and choice.

\section{Who pays?}

Although estimates of the cost of egg freezing vary considerably, the general price range is US\$10,000-\$15,000 per cycle. ${ }^{19}$ One of the factors that can affect the total cost is the quantity of drugs needed to achieve sufficient ovarian stimulation during the egg-retrieval process. In addition, prices can vary depending on the geographic location of the clinic. A separate expense beyond the retrieval and cryopreservation of a woman's eggs is their safe storage. Storage fees are usually several hundred dollars per year. Memorial Sloan Kettering Cancer Center in New York City quotes a price of $\$ 900$ per year for egg storage. ${ }^{20}$ In the USA, health insurance does not pay for "social" egg freezing, and rarely pays for any aspect of the egg-freezing process when done for medical reasons, although there are some exceptions and some movement to change that practice.

When pursued for nonmedical reasons, egg freezing is generally an out-of-pocket expense that falls on the shoulders of women themselves. By some reports in the popular media, it is not uncommon for a woman's parents to provide financial assistance for her egg freezing. ${ }^{21}$ Fertility clinics typically offer financing plans for egg freezing and for the subsequent IVF process when and if a woman chooses to attempt fertilization and implantation. At a minimum, clinics counsel patients on how to navigate the expense. Extend Fertility, an egg-freezing company that partners with nine US fertility clinics, advises on its website that some employers may offer a flexible spending plan that allows employees to set aside pretax dollars to be used for any medical expenses. ${ }^{22}$ Reproductive Biology Associates in Atlanta offers multiIVF-cycle discount packages and a refund guarantee. ${ }^{23}$

Recently, Facebook and Apple made headlines by offering to give female employees $\$ 20,000$ of egg-freezing benefits. ${ }^{24}$ Public reaction to this news was mixed, especially among women. Some viewed the development positively as a forward-thinking practice that would give greater flexibility and peace of mind to young female employees. Others were skeptical that women would be the true beneficiaries, arguing that it would create implicit pressure to partake in egg freezing and delay motherhood in order to demonstrate seriousness and dedication to the workplace. ${ }^{25,26}$ The significance of the Facebook and Apple offers is largely symbolic, as most companies are not likely to provide this kind of benefit to their employees in the near future.

As noted, even when egg freezing is done for medical reasons, health insurance plans in the USA generally do not provide coverage for it. ${ }^{27,28}$ Whatever a woman's motivation for pursuing egg freezing, insurers regard the procedure as elective and treat it as such. There is some anecdotal evidence that patients have been able to convince their insurance companies to cover fertility preservation by billing the treatment under a primary diagnosis of cancer. ${ }^{29}$ However, the extent to which this practice is customary is not known. Ascertaining what aspects of fertility preservation might be covered by insurance often requires the assistance of a financial specialist at the fertility center and strong personal advocacy.

Some private programs offer financial assistance to women diagnosed with cancer who seek to preserve their fertility. LIVESTRONG Fertility is a private foundation that aims to "increase access to fertility preservation services and treatments for qualified women who are diagnosed with cancer during their reproductive years." ${ }^{30}$ Ferring Pharmaceuticals also runs a program called Heartbeat that offers "select fertility medications at no cost" to women diagnosed with cancer. ${ }^{31}$

Some scholars have argued that fertility-preservation treatments for cancer patients ought to be covered by health insurance plans, just as reconstructive breast surgery is 
covered after mastectomy. Campo-Engelstein challenges the designation of infertility treatments and fertility-preservation techniques as "elective" procedures, given the centrality of reproductive capacity to human health and well-being. ${ }^{29}$ She also notes the inconsistency and unfairness of covering some elective procedures and not others:

[I]t is time for insurance companies to stop relegating ART to a separate realm outside of "real" health care, especially when they cover treatment for conditions that could also be perceived as elective. The fact that insurance companies have begun covering fertility preservation treatment for cancer patients gives hope that fertility and infertility treatments are finally being taken seriously by insurance companies. Nevertheless, this coverage is done covertly on a case by case basis rather than under a blanket policy, which insinuates that insurance companies are still not ready to publicly assume financial responsibility for iatrogenic infertility. ${ }^{29}$

She suggests that a legal mandate, perhaps modeled after the Women's Health and Cancer Rights Act, which addresses reconstructive surgery after mastectomy, might be the needed impetus for insurance companies to cover iatrogenic infertility more openly and consistently:

Such a mandate would not only symbolize the importance of fertility preservation treatment and the severity of infertility as a disease but also open the door for more discussions between patients and providers about fertility preservation treatment. Furthermore, a mandate would provide greater access to patients from lower socioeconomic statuses, to patients without insurance, and/or to patients who do not have patient advocates to help them secure funding for this technology. ${ }^{29,32}$

For the time being, only 15 states in the USA require insurance coverage for infertility treatment. One of them, New Jersey, explicitly excludes coverage for cryopreservation. ${ }^{33}$ By contrast, the UK offers coverage for infertility treatment through its National Health Service, including egg freezing for medical reasons. ${ }^{34}$ Israel also subsidizes infertility treatments. As early as 2009, the Israel National Bioethics Council (INBC) recommended permitting egg freezing for disease as well as age-related fertility decline. ${ }^{35}$

Standards for how different countries pay for infertility treatments and for egg freezing are in flux as attitudes about egg freezing evolve. Generally speaking, however, European countries are more generous in their subsidization of infertility treatment and fertility preservation than the USA.

\section{Arguments for and against - ethical perspectives}

When infertility is understood to be an illness, a deprivation of normal bodily functioning that warrants medical intervention, arguments for infertility treatments and for fertility preservation generally rest on the same justification: it is the business of medicine to heal and to promote healthy bodily function. In other words, egg freezing for a woman diagnosed with cancer is justified by the same reasons that support the use of IVF for a woman with blocked fallopian tubes: both take what is broken and attempt to make it whole. Notwithstanding American insurance companies' reluctance to provide adequate coverage for infertility treatments, gone are the days when infertility was judged at the outset to be an unchangeable condition, something to be endured and accepted.

Controversy more commonly arises from differing interpretations of infertility. Should it matter, morally, if the infertility is caused by illness or age? Should it matter if a woman anticipates infertility in the future but does not experience it in the present, and seeks preventive steps to address it preemptively? Just as the line between therapy and enhancement has been difficult to draw in bioethics debates, so too is the line between medical and nonmedical reasons for egg freezing proving blurry at best. In fact, we are witnessing the subtle migration of age-related fertility loss from being thought of as a nonmedical reason to being seen as a medical reason for egg freezing. Under this emerging view, the 30-year-old who has the foresight to freeze her eggs before experiencing any sign of infertility is now not engaging in a nonessential elective procedure, but undertaking "preventive medicine".

As shared cultural understandings of a normative context for human reproduction unravel, what persists as morally salient is the individual choosing and investing those choices with significance. Arguments in favor of social egg freezing generally support the prerogative of individual choice; arguments opposed to it tend to hold onto some larger normative framework beyond what the individual invests with meaning. Whether that larger framework is a vision of the medical profession's responsibility or the government's responsibility to protect individuals from exploitation, a religiously inspired respect for embodied limits, or a feminist focus on social structures that constrain women's well-being, arguments opposed to egg freezing for any reason have to find their justification somewhere outside of a narrowly construed individual autonomy.

Many of the ethical arguments in favor of egg freezing for nonmedical reasons focus on the liberating potential of 
egg freezing for women. As such, they are reminiscent of Firestone's imaginative vision of extracorporeal gestation and its potential to free women from the constraints of biology and time ${ }^{36}$ If biological difference is the root of inequality between men and women, then egg freezing can help level the playing field by lengthening the time during which a woman can become pregnant. Savulescu and Imogen ${ }^{37}$ and Goold and Savulescu ${ }^{38}$ have argued that egg freezing for nonmedical reasons promotes sex equality. Among its many benefits, egg freezing promotes equal participation in employment, equal participation in educational endeavors, and a more equal amount of time to find a partner. Other benefits include more time to become emotionally and psychologically ready to be a parent, something appreciated by men and women alike. Goold and Savulescu embrace egg freezing as a "secondary strategy" to challenging social structures that disadvantage women: "[W]hen discriminatory features of society are changed, [egg freezing] may no longer be necessary. But in the meantime, in our view, it empowers women". ${ }^{38}$ In this way, egg freezing serves as something akin to "reproductive affirmative action". ${ }^{38}$

Mertes similarly appreciates the potential of egg freezing to loosen the constraints of biology and time. ${ }^{39}$ She notes the benefits of being an older parent, including benefits for the child, and suggests that restructuring society to encourage childbearing at a younger age is not necessarily desirable. Setting aside issues of sex discrimination in the workplace, women may have good reasons to prefer to postpone childbearing. Unfortunately, the "perfect" biological time for reproducing - thought to be between the ages of 25 and 35 years for women - may not align with a woman's preferences. Rather than viewing egg freezing as the alternative to reproducing at a younger age, Mertes argues it should be viewed as the alternative to using a donor egg when agerelated infertility is later encountered. From this perspective, egg freezing becomes a form of prudent preventive medicine, especially if undertaken in time for its likelihood of success to be high.

Other arguments in favor of egg freezing for nonmedical reasons focus not on sex equality per se but on the importance of informed consent and the principles of autonomy and beneficence that underlie it. Rybak and Lieman point out that what society deems to be an acceptable success and safety rate for any new medical technology is ultimately arbitrary; there is no bright line beyond which we can be sure that a risk-benefit ratio is universally acceptable. In their view, the proper place to weigh up risks and benefits is the informed consent process, which respects individual decision making and individuals' values. ${ }^{40}$ Given what the authors consider to be a rush to adopt ICSI, the slow acceptance of egg freezing by comparison looks like evidence of a sexist double standard. Without throwing caution to the wind, Rybak and Lieman put their faith in individual choice:

[F]or the informed woman seeking procreative liberty via greater control over her reproductive destiny, the considerations of autonomy and beneficence override those of commercialization, deleterious change and exploitation. ${ }^{40}$

Arguments that rely heavily or even exclusively on the value of individual autonomy to justify egg freezing do not necessarily need to address the question of whether there are morally significant differences between medical and nonmedical reasons for egg freezing. If what matters, ultimately, is individual preference and choice, then background motivations would seem to fall away as unimportant. Nevertheless, recognizing that autonomy is not an absolute value, scholars do pay attention to the reasons for egg freezing. Goold and Savulescu ${ }^{38}$ argue that the harm of infertility is the same regardless of whether it is caused by illness or age. Petropanagos agrees, and elaborates their argument. She looks more explicitly at the causes of age-related infertility, and argues that women should not be blamed for their "perpetual postponement" of pregnancy. She brings a feminist understanding of relational autonomy to her assessment: "Unlike traditional accounts of autonomy, feminists' accounts of autonomy require an explicit recognition that autonomy is both defined and pursued in a social context." ${ }^{\prime 4}$ Given the context of sexist social structures, including professional norms, the "choice" to delay childbearing may not be as voluntary as it seems. She concludes:

If we continue to allow disease-related egg freezing, then we ought to also allow age-related egg freezing, given the patriarchal context of women's reproductive choices. Until the sexist social structures that shape and confine women's reproductive choices change, many women may continue to find their lives unfolding in ways that result in delayed motherhood. ${ }^{4}$

Many feminist scholars pay attention to the social context of egg freezing, especially if they deem the root of sexual inequality to be in the social construction of gender. However, recognizing that women's reproductive decision making is socially constructed and constrained can lead to divergent conclusions. Some, like Petropanagos, ${ }^{4}$ see the compensatory value of egg freezing. Others wonder more skeptically whether egg freezing may do more harm than good. It is not 
only that egg freezing is viewed by these feminists as a small and short-term fix that leaves sexist structures fundamentally unchanged. ${ }^{25,41,42}$ Many would concede, after all, that "reproductive affirmative action" can work in tandem with largerscale efforts at social reform. The problem, according to this view, is that opening the door to the option creates a pressure to use it. As philosopher Michael Sandel once claimed in a discussion of the hypothetical problems created by genetic engineering, offering the option to genetically engineer one's children could engender a sense of hyperresponsibility in parents for their offspring's traits. ${ }^{43}$ In an environment where intervening genetically prebirth became the norm, it would no longer be a misfortune if your child was short or nearsighted or hemophiliac; it would now be the parents' fault for not taking advantage of an available technology proactively. Similarly, as pointed out when Apple and Facebook made their generous offers, the option to freeze eggs might quickly become an obligation to freeze eggs, a way to demonstrate one's seriousness about one's career, or a way to avoid selfblame. ${ }^{19}$ In fact, there is already evidence from an empirical study of women's motivations that a major impetus for eggfreezing is the avoidance of self-blame: "I want to know I did everything I could and not blame myself later". ${ }^{14}$

Arguments that oppose egg freezing, or at least find it sufficiently problematic to justify restricting in some way, often identify harms believed to be significant enough to warrant limiting individual autonomy. First among these would be harms to women themselves, including raising false hopes and commercial exploitation. Given the very low success rates of egg freezing for women in their 40s, the potential for commercial exploitation undeniably exists. Second would be harms to children. Available data suggest that children born from egg freezing and IVF do not experience any statistically significant physical or developmental harm, although long-term studies are not yet available. Some ethicists have also raised the issue of the harm to children caused by having older or even elderly parents. According to philosopher Onora O'Neill, "there is a difference between the misfortune of the early death of a young parent, and actively setting up a situation in which the likelihood of death while the child is young is increased". ${ }^{44}$ Both the low success rates of egg freezing for older women and concerns about setting up a situation where the early death of a parent is more likely could warrant imposing age limits for egg freezing and/or IVF. Finally, there are social harms to consider, including exacerbating a class divide based on who can afford to access the technology and reinforcing a "bioessentialist" understanding of family that requires genetic connection between parent and child. ${ }^{41}$
Religious perspectives on egg freezing run the gamut from enthusiastic embrace to unequivocal opposition. A full exploration of these diverse views is beyond the scope of this review. In brief, some of the most positive appraisals of egg freezing take their inspiration from an embrace of pronatalism and the duty to heal. ${ }^{45,46}$ Some of the most critical appraisals reject the secular framework that views reproduction as a personal project to which one has a right. ${ }^{47}$ Catholic tradition, for example, does not view procreation as a process of manufacture, infused only with the significance humans choose to give it. Respect for embodied limitations, the "givenness" and sanctity of created life, including aging and mortality, is fundamental to this worldview, and it forms the basis of the Catholic view of procreation as inseparable from married sexual intercourse.

Most of the relevant literature on egg freezing brackets or compartmentalizes religious worldviews out of respect for pluralism. According to the European Society of Human Reproduction and Embryology:

In a secular debate, the problem with arguing from views about 'the good life' is that they rest on religious or naturalistic presuppositions that not all participants necessarily share. [F] ertility specialists should leave it to the women themselves to make their own informed decisions. ${ }^{15}$

This approach is in line with respect for individual autonomy. However, there is some irony in the fact that certain ideas of "the good life" enjoy widespread acceptance - to the point of being no longer seen as specific ideas of the good life - while others do not. For example, the presumption that a genetic connection to one's child is a neutral "good" is widely accepted. The idea that reproduction should take place during the years of naturally occurring female fertility does not currently enjoy similar agreement.

Egg freezing, not unlike the birth-control pill in the 1960s and 1970s, has become the site of fascinating ethical debate and a crucible of contested worldviews. The impact it will have on women's reproductive autonomy, the family, and the workplace remains to be seen. Popular books, such as Motherhood Rescheduled, have chronicled women's decision making about egg freezing, but a great deal more information about both clinical and cultural outcomes needs to be gathered before an ethical consensus about egg freezing for nonmedical reasons can be reached. ${ }^{48,49}$

\section{Route to professional acceptance; policy}

Without a scholarly consensus about nonmedical egg freezing, and without the ASRM's explicit endorsement of nonmedical 
egg freezing, perhaps the most important policy recommendation may be to encourage egg freezing only when the procedure is most likely to succeed. ${ }^{50}$ Currently, the average reported age of women who freeze their eggs is 38 years, but that may be too late. By 38 years, the quality of a woman's eggs is already in decline. Unfortunately, studies have shown that women do not consider egg freezing until precisely that age: late 30 s or older. The ideal age for egg freezing is reportedly 30-35 years, but these younger women tend not to consider egg freezing because they believe they have plenty of time and/or they underestimate their natural fertility decline after age 35 years. Therefore, according to currently available knowledge about utilization, there is a troubling mismatch between patient demand and optimal outcome.

At a minimum, if egg freezing for nonmedical reasons is going to proceed in clinics around the world, women need to be fully informed about the success rates, and success rates need to be broken down by age-groups. Mertes and Pennings additionally recommend that "success rate" be more carefully and transparently defined for patients: does it mean clinical pregnancy rate, birth rate, or something else? ${ }^{50}$ Much greater consistency and thoroughness in reporting would yield better and more generalizable data.

It is reasonable to require better informed consent, truthful advertising of success rates, and more rigorous long-term studies of outcomes, including studies of children born through egg freezing. While egg freezing for women aged over 38 years need not be banned outright, there should be candid disclaimers about the low probability of successful thawing, fertilization, implantation, and live birth. Without this transparency and truthful disclosure, egg freezing for women aged over 40 years becomes the ultimate snake oil: an expensive procedure that is not much more than an empty promise.

The deeper ethical questions raised by "social" or nonmedical egg freezing do not yield to easy answers. Does nonmedical egg freezing promote sex equality or undermine it? Does it enhance human health and well-being, or does it encourage a harmful artificiality? Answering these questions depends largely on one's prior normative frameworks, including how one understands the nature and origin of sexual inequality and the purpose and limits of medical technology. In lieu of broad consensus about these matters, the best policy is to honor the principle of autonomy while insisting on better information about utilization and outcomes. As a start, in the USA, the Centers for Disease Control and Prevention could begin compiling data on egg freezing as part of its annual assisted reproductive technology report. ${ }^{51}$

\section{Disclosure}

The author reports no conflicts of interest in this work.

\section{References}

1. Practice Committees of the American Society for Reproductive Medicine, Society for Assisted Reproductive Technology. Mature oocyte cryopreservation: a guideline. Fertil Steril. 2013;99(1):37-43.

2. Johnston J, Zoll M. Is freezing your eggs dangerous? A primer. The New Republic; 2014. Available from: http://www.newrepublic.com/ article/120077/dangers-and-realities-egg-freezing. Accessed May 21, 2015.

3. Waldby C. 'Banking time': egg freezing and the negotiation of future fertility. Cult Health Sex. 2015;17(4):470-482.

4. Petropanagos A. Reproductive 'choice' and egg freezing. In: Woodruff TK, Zoloth L, Campo-Engelstein L, Rodriguez S, editors. Oncofertility: Ethical, Legal, Social and Medical Perspectives. New York: Springer; 2010:223-235.

5. Cil AP, Bang H, Oktay K. Age-specific probability of live birth with oocyte cryopreservation: an individual patient data meta-analysis. Fertil Steril. 2013;100(2):492-499. e3.

6. Rudick B, Opper N, Paulson R, Benikson K, Chung K. The status of oocyte cryopreservation in the United States. Fertil Steril. 2010;94(7): 2642-2646.

7. Quaas AM, Melamed A, Chung K, Bendikson KA, Paulson RJ. Egg banking in the United States: current status of commercially available cryopreserved oocytes. Fertil Steril. 2013;99(3):827-831.

8. Cobo A, Diaz C. Clinical application of oocyte vitrification: a systematic review and meta-analysis of randomized controlled trials. Fertil Steril. 2011;96(2):277-285.

9. Grifo JA, Noyes N. Delivery rate using cryopreserved oocytes is comparable to conventional in vitro fertilization using fresh oocytes: potential fertility preservation for female cancer patients. Fertil Steril. 2010;93(2):391-396.

10. Loren AW, Mangu PB, Beck LN, et al. Fertility preservation for patients with cancer: American Society of Clinical Oncology clinical practice guideline update. J Clin Oncol. 2013;31(19):2500-2510.

11. National Cancer Institute Surveillance, Epidemiology, and End Results Program. SEER stat fact sheets: breast cancer. Available from: http:// seer.cancer.gov/statfacts/html/breast.html. Accessed May 26, 2015.

12. Ethics Committee of the American Society for Reproductive Medicine. Fertility preservation and reproduction in patients facing gonadotoxic therapies: a committee opinion. Fertil Steril. 2013;100(5): 1224-1231.

13. Fernbach A, Lockart B, Armus C, et al. Evidence-based recommendations for fertility preservation options for inclusion in treatment protocols for pediatric and adolescent patients diagnosed with cancer. J Pediatr Oncol Nurs. 2014;31(4):211-222.

14. Witkin G, Tran A, Lee JA, Schuman L, Grunfeld L, Knopman JM. What makes a woman freeze: the impetus behind patients' desires to undergo elective oocyte cryopreservation. Fertil Steril. 2013;100(3):S24.

15. Dondorp W, de Wert G, Pennings G, et al. Oocyte cryopreservation for age-related fertility loss. Hum Reprod. 2012;27(5):1231-1237.

16. Matthews TJ, Hamilton BE. First births to older women continue to rise. NCHS Data Brief. 2014;(152):1-8.

17. Stoop D, Cobo A, Silber S. Fertility preservation for age-related fertility decline. Lancet. 2014;384(9950):1311-1319.

18. Ethics Committee of the American Society for Reproductive Medicine. Access to fertility treatments by gays, lesbians, and unmarried persons: a committee opinion. Fertil Steril. 2013;100(6):1524-1527.

19. Mohapatra S. Using egg freezing to extend the biological clock: fertility insurance or false hope? Harv Law Policy Rev. 2014;8(382):381-411.

20. Memorial Sloan Kettering Cancer Center. Fertility preservation: options for women who are starting cancer treatment. Available from: https:// www.mskcc.org/cancer-care/patient-education/cancer-and-fertilityinformation-women. Accessed May 18, 2015. 
21. Gootman E. So eager for grandchildren, they're paying the egg-freezing clinic. 2012. Available from: http://nyti.ms/18FRjDb. Accessed May 18, 2015.

22. Extend Fertility. Egg freezing process. Available from: http://www. extendfertility.com/egg-freezing-process. Accessed May 19, 2015.

23. Reproductive Biology Associates. Financial information/insurance. Available from: http://www.rba-online.com/ivf/index.php?FinancialInformation-Insurance-24. Accessed May 19, 2015.

24. Rooney B. Facebook, Apple pay to freeze employees' eggs. 2014. Available from: http://money.cnn.com/2014/10/14/news/companies/ facebook-apple-egg-freeze. Accessed May 19, 2015.

25. Mead R. Cold comfort: tech jobs and egg freezing. 2014. Available from: http://www.newyorker.com/news/daily-comment/facebookapple-egg-freezing-benefits. Accessed May 19, 2015.

26. Almeling R, Radin J, Richardson SS. Egg-freezing a better deal for companies than for women. 2014. Available from: http:/www.cnn. com/2014/10/20/opinion/almeling-radin-richardson-egg-freezing. Accessed May 19, 2015

27. Andrews M. Few employers cover egg freezing for women with cancer. 2014. Available from: http://www.npr.org/sections/healthshots/2014/12/16/371009911/few-employers-cover-egg-freezing-forwomen-with-cancer. Accessed May 19, 2015.

28. Andrews M. Few women have coverage for egg freezing. 2014. Available from: http://kaiserhealthnews.org/news/few-women-havecoverage-for-egg-freezing. Accessed May 19, 2015.

29. Campo-Engelstein L. For the sake of consistency and fairness: why insurance companies should cover fertility preservation treatment. In: Woodruff TK, Zoloth L, Campo-Engelstein L, Rodriguez S, editors. Oncofertility: Ethical, Legal, Social and Medical Perspectives. New York: Springer; 2010:381-389.

30. LiveStrong Fertility. Criteria and application for women [application form]. Available from: http://images.livestrong.org/downloads/we-canhelp/LF_App_Women_FINAL_v9.pdf?_ga=1.237964992.190229653 1.1415306052. Accessed May 20, 2015.

31. Ferring Reproductive Health. The Heart Beat Program. 2014. Available from: https://www.ferringfertility.com/savings/heartbeat. Accessed May 20, 2015.

32. United States Department of Labor. Employee Benefits Security Administration: Your rights after a mastectomy. Available from: http:// www.dol.gov/ebsa/publications/whcra.html. Accessed May 27, 2015.

33. RESOLVE: The National Fertility Organization. Insurance coverage. McLean, VA: RESOLVE; 2015. Available from: http://www.resolve. org/family-building-options/insurance_coverage/state-coverage.html. Accessed May 18, 2015.

34. Human Fertilisation and Embryology Authority. Freezing and storing eggs. 2013. Available from: http://www.hfea.gov.uk/46.html. Accessed May 28, 2015.
35. Shkedi-Rafid S, Hashiloni-Dolev Y. Egg freezing for age-related fertility decline: preventive medicine or further medicalization of reproduction? Analyzing the new Israeli policy. Fertil Steril. 2011;96(2):291-294.

36. Firestone S. The Dialectic of Sex: The Case for Feminist Revolution. New York: Morrow; 1970.

37. Savulescu J, Imogen G. Freezing eggs for lifestyle reasons. Am J Bioeth. 2008;8(6):32-35.

38. Goold I, Savulescu J. In favour of freezing eggs for non-medical reasons. Bioethics. 2009;23(1):47-58.

39. Mertes H. The portrayal of healthy women requesting oocyte cryopreservation. Facts Views Vis Obgyn. 2013;5(2):141-146.

40. Rybak EA, Lieman HJ. Egg freezing, procreative liberty, and ICSI: the double standards confronting elective self-donation of oocytes. Fertil Steril. 2009;92(5):1509-1512.

41. Cattapan A, Hammond K, Haw J, Tarasoff LA. Breaking the ice: young feminist scholars of reproductive politics reflect on egg freezing. Int $J$ Fem Approaches Bioeth. 2014;7(2):236-247.

42. Harwood K. Egg freezing: a breakthrough for reproductive autonomy? Bioethics. 2009;23(1):39-46.

43. Sandel MJ. The case against perfection: what's wrong with designer children, bionic athletes, and genetic engineering. Atl Mon. 2004;293(3):50-54, 56-60, 62.

44. O'Neill O. Autonomy and Trust in Bioethics. Cambridge: Cambridge University Press; 2000.

45. Kahn SM. Reproducing Jews: A Cultural Account of Assisted Conception in Israel. Durham: Duke University Press; 2000.

46. Zoloth L. Jewish perspectives on oncofertility: the complexities of tradition. In: WoodruffTK, Zoloth L, Campo-Engelstein L, Rodriguez S, editors. Oncofertility: Ethical, Legal, Social and Medical Perspectives. New York: Springer; 2010:307-317.

47. Lauritzen P. Technology and wholeness: oncofertility and Catholic tradition. In: WoodruffTK, Zoloth L, Campo-Engelstein L, Rodriguez S, editors. Oncofertility: Ethical, Legal, Social and Medical Perspectives. New York: Springer; 2010:295-306.

48. Richards SE. Motherhood, Rescheduled: The New Frontier of Egg Freezing and the Women Who Tried It. New York: Simon \& Schuster; 2013.

49. Baldwin K, Culley L, Hudson N, Mitchell H. Reproductive technology and the life course: current debates and research in social egg freezing. Human Fertil. 2014;17(3):170-179.

50. Mertes H, Pennings G. Social egg freezing: for better not for worse. Reprod Biomed Online. 2011;23(7):824-829.

51. Centers for Disease Control and Prevention. National ART success rates. 2012. Available from: http://nccd.cdc.gov/DRH_ART/Apps/ NationalSummaryReport.aspx. Accessed May 31, 2015.
Medicolegal and Bioethics

\section{Publish your work in this journal}

Medicolegal and Bioethics is an international, peer-reviewed, open access journal exploring the application of law to medical and drug research and practice and the related ethical and moral considerations. The journal is characterized by the rapid reporting of reviews, case reports, guidelines and consensus statements, original research

\section{Dovepress}

and surveys. The manuscript management system is completely online and includes a very quick and fair peer-review system. Visit http://www.dovepress.com/testimonials.php to read real quotes from published authors. 